\title{
VLBI and Doppler tracking of spacecraft for planetary atmospheric studies
}

\section{T.M. Bocanegra-Bahamón ${ }^{* 1,2,3}$, L.I. Gurvits ${ }^{1,2}$, G. Molera Calvés ${ }^{4}$, G. Cimò̀ ${ }^{1,5}$, D.A. Duev $^{6}$ and S. Pogrebenko ${ }^{2}$.}

1. Department of Astrodynamics and Space Missions, Delft University of Technology, The Netherlands.

2. Joint Institute for VLBI in Europe, The Netherlands.

3. Shanghai Astronomical Observatory, China.

4. Finnish Geospatial Research Institute, Finland.

5. Netherlands Institute for Radio Astronomy, The Netherlands.

6. California Institute of Technology, USA.

E-mail: t.m.bocanegrabahamonetudelft.nl

The Planetary Radio Interferometry and Doppler Experiment (PRIDE) is a technique that can enhance the science return of planetary missions. By shadow tracking the spacecraft signal using radio telescopes from VLBI networks, the PRIDE technique provides precise open-loop Doppler and near-field VLBI observables [1,2] to find the radial velocity of the spacecraft and its position in the plane of the sky. This information is not only important for navigation, but it can also be used for many science applications. One such case is the study of planetary atmospheres by means of radio occultation experiments. The application of PRIDE for atmospheric studies has been demonstrated by observing ESA's Venus Express (VEX) during multiple Venus occultation events [3]. From these observations density, temperature and pressure profiles of Venus were derived to characterize the planet's ionosphere and neutral atmosphere. The noise budget of the observations indicated that the quality of the detections are comparable to those of NASA's and ESA's deep space networks [2]. With PRIDE, making use of open-loop Doppler data, EVN stations were able to sound deeper layers of Venus' thick atmosphere when compared to closedloop Doppler data provided by ESA's New Norcia.

14th European VLBI Network Symposium \& Users Meeting (EVN 2018)

8-11 October 2018

Granada, Spain

${ }^{*}$ Speaker. 


\section{Introduction}

Electromagnetic emission is an indispensable means of communication with interplanetary spacecraft in their journeys through the Solar System. The transmission and reception of radio signals to and from interplanetary spacecraft have three main functions. The first is the generation of radiometric data (e.g., Doppler, ranging and interferometric data) to enable the precise determination of the spacecraft's state vector (i.e., position and velocity) along its trajectory. The second is for telecommunication purposes, sending commands to the spacecraft on the uplink and transmitting telemetry (i.e. engineering and science data produced by the instruments onboard the spacecraft) on the downlink. The third function is to conduct scientific research by analyzing the different effects that the presence of the planetary bodies and interplanetary medium has on the spacecraft signal, as it propagates back to the Earth. This functionality is usually refer to as the mission's radio science experiment.

The two first functions are vital for the mission, while the third is not necessarily. For this reason, these are the main priorities of the deep space networks such as NASA's Deep Space Network (DSN) and ESA's Estrack: to support navigation and to control and monitor the status of the spacecraft [8]. The third function is generally implemented using the deep space network facilities as well. With the Planetary Radio Interferometry and Doppler Experiment (PRIDE), we present an alternative to using the deep space networks for this function - radio science experiments - focusing primarily on one application, radio occultation studies.

\section{The Planetary Radio Interferometry and Doppler Experiment (PRIDE)}

The PRIDE technique is an experimental setup that makes use of the onboard spacecraft transponder and Earth-based radio telescope networks, in order to provide (1) radial velocity and (2) angular position measurements, to support radio science experiments [1, 5, 7, 2]. This is done by performing precise Doppler tracking of the spacecraft carrier signal with multiple radio telescopes on Earth. A wideband spectral analysis is conducted to extract the topocentric Doppler shift of the carrier signal, due to the relative motion of the transmitter and receiver, at each station. Subsequently, these signals are cross-correlated using a near-field Very Long Baseline Interferometry (VLBI) model in the phase-referencing mode. The objective of the near-field VLBI model is to calculate the a priori difference in the arrival time of the curved wave front of the signal, at every two stations of the observing array, in order to achieve a coherent response (the so-called 'interferometric fringes') by cross-correlating the signals. This is actually the fundamental principle behind radio interferometry [11], but taking into account that the source of the radio signal (i.e. the spacecraft) is at a finite distance from the receivers. In the case of VLBI, the receivers are located at very long distances from each other, and therefore, independent timing (local oscillators) and signal recording systems are required. With the information from the near-field modeled delays and the phase and frequency of the resulting interference fringe pattern, the angular position of the radio source can be determined. The latter is achieved in the so-called phase-referencing mode [11]. In this mode, other radio sources that are angularly close-by to the target source $(\sim<2 \mathrm{deg})$ are used to calibrate the phase of the spacecraft signal. The calibrator sources are typically chosen to be natural sources whose absolute positions are known very accurately (in the level of tens of 
$\mu$ as). This summarizes briefly the methodology used by PRIDE to derive the radial velocity and angular position of the target spacecraft.

\section{PRIDE performance}

In order to benchmark the technique with respect to the use of the traditional deep space networks for radio science investigations, a noise budget of the Doppler detections obtained with the PRIDE was performed [2]. As a test case a tracking experiment of the European Space Agency (ESA) Mars Express (MEX) was used, during which the spacecraft performed a fly-by around Mars' moon Phobos in December 2013. In this experiment, 31 radio telescopes (including 13 telescopes from the European VLBI Network (EVN)) around the world participated for a total of 26 hours of continued tracking of MEX. In order to determine the quality of the PRIDE Doppler detections, the random errors introduced by the instrumentation and by the propagation of the signal through the interplanetary media were analyzed. This analysis quantified the contribution of the different noise sources to the total Doppler residuals. This information was used to provide a noise budget of the observations. It was demonstrated that the residual frequencies obtained with PRIDE Doppler detections are at the same noise levels as those of standard Doppler detections obtained with NASA's and ESA's deep space network antennas $(\sim 2 \mathrm{mHz})$. Figure 1 shows as an example the frequency residuals found with the $25 \mathrm{~m}$ antenna of the Very Long Baseline Array (VLBA) at Kitt Peak (Kp) and the residuals of the 70 m DSS- 63 and DSS-14 antennas. In this case, the median value of the difference between the fit of Kp and the fit of DSS-63 and DSS-14, respectively, remains below $1 \mathrm{mHz}$ for an integration time of $10 \mathrm{~s}$.

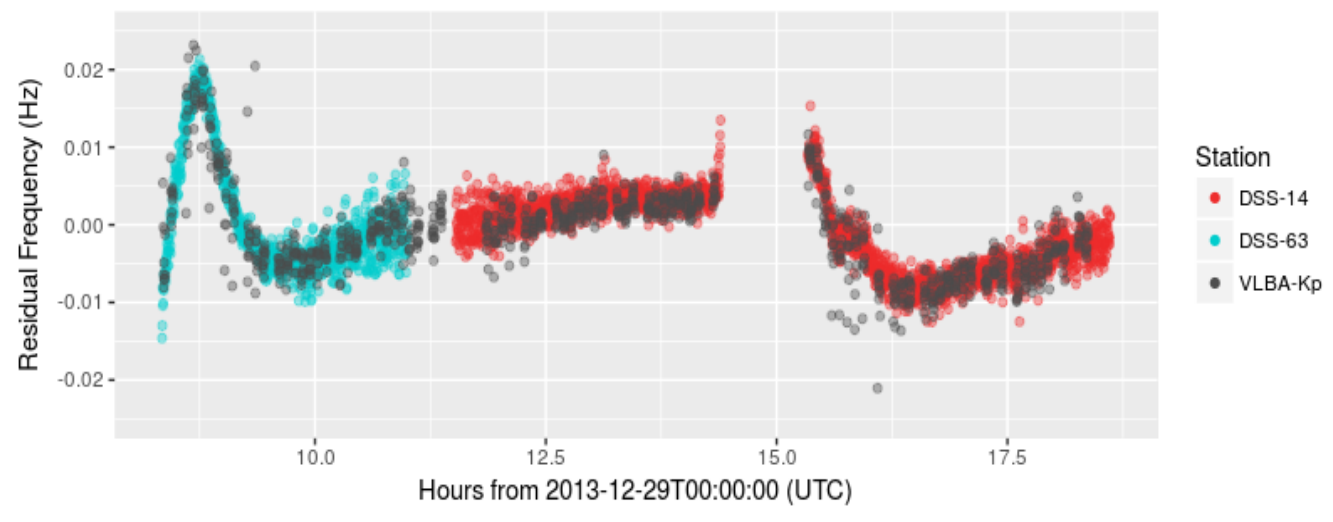

Figure 1: Comparison of the Doppler residuals obtained with VLBA-Kp (in black), DSS-63 (in blue), and DSS-14 (in red). The median value of the difference between the fit of VLBA-Kp and the fit of DSS-63 and DSS-14, respectively, remains below $1 \mathrm{mHz}$, for an integration time of $10 \mathrm{~s}$ [2].

\section{PRIDE for planetary atmospheric studies}

Having validated the methodology used in PRIDE, its applicability and performance for conducting planetary atmospheric studies was investigated by means of radio occultation experiments [3]. When a spacecraft is orbiting a planet the signal of the spacecraft can get occulted by the 
celestial body as seen from the ground stations on Earth. If the planet has an atmosphere, before the signal gets completely occulted it will get refracted through the planet's atmosphere, and the resulting frequency changes in the carrier signal will be detected by the receiving ground stations. By performing spectral analysis of the received signal and with precise information of the position and velocity of the spacecraft, the physical properties of the section of the atmosphere which the spacecraft signal has sounded can be derived (e.g., [10, 9]).

This particular application of PRIDE has been assessed by observing ESA's Venus Express (VEX) during multiple Venus occultation events [3]. From these observing sessions density, temperature and pressure profiles of the neutral atmosphere of Venus have been derived. With this VEX test case, it has been demonstrated that the PRIDE setup and processing pipeline is very well suited for radio occultation experiments of planetary bodies. The noise budget of the observations obtained with the VLBI stations indicated that the uncertainties in the Doppler detections are in the same order of magnitude of those obtained with NASA's Deep Space Network (DSN) and ESA's Estrack. For instance, uncertainties in the order of $2 \mathrm{mHz}$ in the Doppler residuals result in uncertainties of $\sim 0.01 \mathrm{~K}$ in the temperature profiles of Venus' neutral atmosphere. These results correspond to those reported in previous Venus radio occultation experiments involving DSN stations (e.g., [4]). We have also demonstrated that with PRIDE, making use of open-loop Doppler data, VLBI stations were able to sound deeper layers of Venus' thick atmosphere when compared to the closed-loop Doppler data provided by Estrack's New Norcia, as shown in Figure 2. With the wideband spectral analysis of PRIDE, we showed that even with small dish antennas, such as the 12-m AuScope's Katherine (Australia Northern Territory), the spacecraft signal can be detected below Venus' clouds layer.
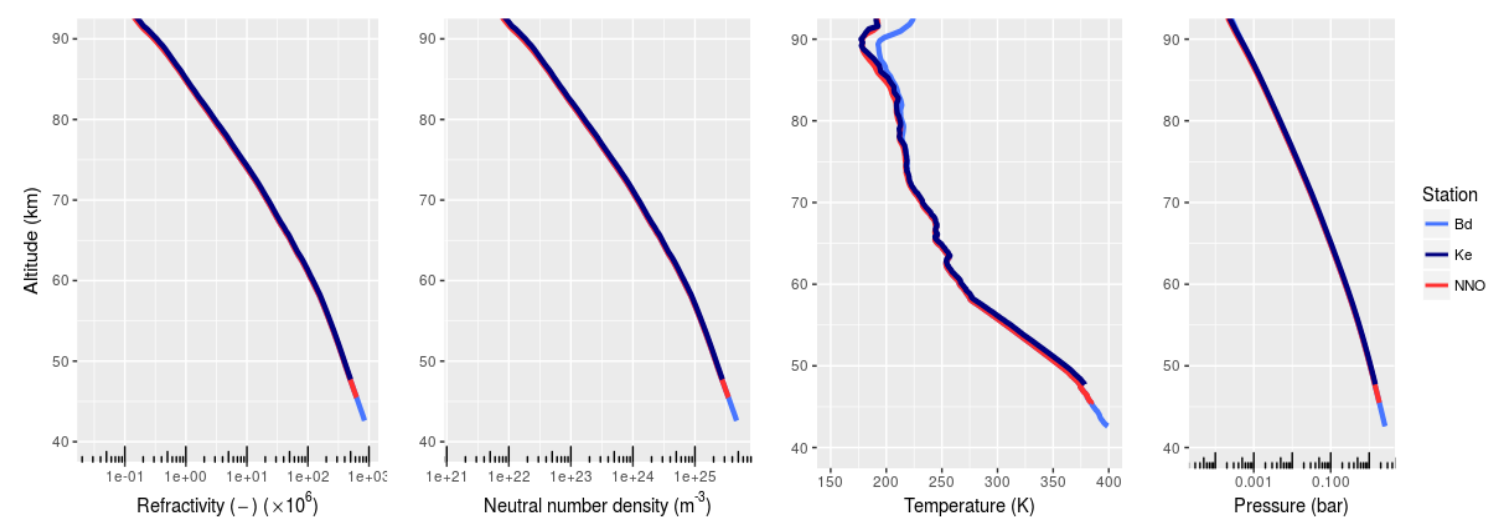

Figure 2: Refractivity, neutral number density, temperature and pressure profiles of Venus atmosphere. The profiles corresponding to the 32-m Bd and 12-m Ke were derived from the open loop Doppler data obtained with the PRIDE setup, and the profiles of the 35-m NNO were derived using the frequency residuals obtained from ESA's PSA, corresponding to close loop Doppler tracking data. Despite the fact that both NNO and Bd have similar antenna dish sizes, with Bd the spacecraft signal is detected down to a lower altitude, due to the fact that the Doppler data obtained with Bd is open loop, while for NNO is closed loop [3].

Radio occultation experiments carried out with PRIDE can exploit the advantage of having access to large radio telescopes from the global VLBI networks, such as the 65-m Tianma (China), 100-m Effelsberg (Germany) or the 305-m Arecibo (Puerto Rico). Additionally, due to the wide 
coverage of the networks, the setup can be optimized to ensure high signal-to-noise (SNR) detections. Since their conception, the hardware and data acquisition software of the DSN and VLBI networks have been developed in close collaboration, and hence their performance is expected to be similar. However, there is a larger number of VLBI radio telescopes than deep space antennas distributed around the world. This allows us to choose those telescopes for which the observing conditions are the best (i.e., highest elevation), while the deep space facilities are limited in this type of operational optimization. The combination of high sensitivity telescopes and wide coverage offers a great opportunity, especially when conducting radio occultation experiments with limited SNR of planets or moons with thick atmospheres.

\section{Conclusions}

Based on the results presented in [2] and [3], it was demonstrated that the spectral analysis used in PRIDE allows the derivation of Doppler observables with very high spectral resolution. Additionally, it provides precise Doppler phase corrections essential to the correct correlation of the spacecraft signal, enabling the precise derivation of angular position corrections to the a priori spacecraft orbit. By demonstrating the performance of PRIDE and providing a noise budget to its Doppler detections, it is shown that the PRIDE technique qualifies as a fully operational radio science instrument. In particular, with the VEX radio occultation test case, it was demonstrated that the PRIDE methodology is suitable for experiments that require a careful detection of highly dynamic signals. It can be concluded that PRIDE, using a wide range of radio telescopes around the world, presents an alternative to using the customary deep space networks for radio science investigations. In particular, when the requests to support multiple deep space missions have conflicting schedules for the nominal agencies' tracking assets, or complementing each other, by enhancing the science return of tracking passes that are not nominally designed for radio science experiments.

PRIDE is one of the eleven instruments selected by ESA for its L-class JUpiter ICy moons Explorer mission (JUICE) mission, to be launched in 2022. The work presented will be used to plan and optimize the setup of PRIDE to conduct radio occultation experiments to study Jupiter's atmosphere. Additionally, PRIDE is involved in the ESA EnVision M5 design study for an orbiter mission to Venus. The main objective of PRIDE will be to conduct the radio occultation experiments to sound Venus' neutral atmosphere and ionosphere. In its current status the PRIDE technique, through the data processing and analysis methodology developed in $[1,7,2,3]$, is in the capacity of conducting radio occultation experiments with virtually any planetary spacecraft.

\section{Acknowledgements}

This publication has received funding from the European Union's Horizon 2020 research and innovation programme under grant agreement No 730562 [RadioNet].

\section{References}

[1] Duev, D.A., Molera Calves, G., and Pogrebenko, S.V., Gurvits, L.I., Cimò, G. and Bocanegra-Bahamón, T.M.: Spacecraft VLBI and Doppler tracking: algorithms and implementation, Astronomy \& Astrophysics, Vol. 541, 2012. 
[2] Bocanegra-Bahamon, T.M., Molera Calves,G. and Gurvits, L.I., Duev, D.A., Pogrebenko, S.V., Cimò, G. and Dirkx, D. and Rosenblatt, P.: Planetary Radio Interferometry and Doppler Experiment (PRIDE) technique: A test case of the Mars Express Phobos Flyby-II. Doppler tracking: Formulation of observed and computed values, and noise budget, Astronomy \& Astrophysics, 609, A59, 2018.

[3] Bocanegra-Bahamon, T.M., Molera Calves,G. and Gurvits, L.I., Dirkx, D. et al.: Venus Expess radio occultation observed by PRIDE, Accepted in Astronomy \& Astrophysics, 2019.

[4] Tellmann, S. Paetzold M., et al.: Structure of the Venus neutral atmosphere as observed by the Radio Science experiment VeRa on Venus Express, Journal of Geophysical Research: Planets,114,E9,2009.

[5] Duev, D. A., Pogrebenko, S. V., Cimò, G., Molera Calvés, G., Bocanegra-Bahamón, T.M., Gurvits, L.I.: Planetary Radio Interferometry and Doppler Experiment (PRIDE) technique: A test case of the Mars Express Phobos fly-by, Astronomy \& Astrophysics, 593, A34, 2016.

[6] Kliore, A.J., Anderson, J.D., Armstrong, J.W. et al.: Cassini radio science, The Cassini-Huygens Mission, pp. 1-70, 2004.

[7] Molera-Calvés, G., Pogrebenko, S., Cimò, G., et al.: Observations and analysis of phase scintillation of spacecraft signal on the interplanetary plasma Astronomy \& Astrophysics, 564, A4, 2014.

[8] Mudgway, D.J. and Launius, R.: Uplink-Downlink: A History of the Deep Space Network, 1957-1997, 2001.

[9] Pätzold, M., Häusler, B., Bird, M.K., Tellmann, S.: The structure of Venus' middle atmosphere and ionosphere, Nature, 450, 657-660, 2007.

[10] Steffes, P.G., Jenkins, J.M., Austin, R.S. et al.: Radio occultation studies of the Venus atmosphere with the Magellan spacecraft: 1. Experimental description and performance, Icarus, 110, 1, 71-78, 1994.

[11] Thompson, A.R., Moran, J.M., Swenson, G.W.: Interferometry and synthesis in radio astronomy, 2008. 